\title{
Cooperative Downlink Transmission Mode Selection under Limited-Capacity Backhaul
}

\author{
Qian Zhang, Chenyang Yang \\ School of Electronics and Information Engineering, \\ Beihang University, Beijing, China \\ Email: qianzhang@ee.buaa.edu.cn, cyyang@buaa.edu.cn
}

\author{
Andreas F. Molisch \\ Ming Hsieh Department of Electrical Engineering, \\ University of Southern California, Los Angeles, USA \\ Email: molisch@usc.edu
}

\begin{abstract}
Coordinated multi-point transmission (CoMP) has been widely recognized as a promising technique for improving spectral efficiency in future cellular systems. However, it may require high-capacity backhaul links, which can increase expense. In this paper, we study the cooperative transmission method with limited-capacity backhaul. Downlink CoMP transmission requires the exchange of information between base stations, depending on the type of CoMP schemes. Either channel state information (CSI) information of all users (for coordinated beamforming), or both data and CSI (for joint processing) need to be shared among all cooperative base stations. The latter method exhibits better performance, but requires high capacity backhaul links. For this reason, we propose in this paper a transmission mode selection method under capacity constrained backhaul. We start by analyzing the performance of two kinds of CoMP transmission modes: joint processing and coordinated beamforming with various types of user scheduling. Then we propose a closed-form decision rule, which depends on the strongest average channel gains of the users co-scheduled in the same time-frequency resource. Simulation results show that the proposed transmission mode selection outperforms the singlemode CoMP transmission.
\end{abstract}

\section{INTRODUCTION}

Inter-cell interference (ICI) is a bottleneck to achieve high spectrum efficiency of universal frequency reuse cellular networks, especially for multi-input-multi-output (MIMO) systems. Recently, cooperative transmission of base stations (BSs), also known as coordinated multi-point (CoMP) transmission in 3GPP Long-Term Evolution-Advanced (LTE-A) [1], has been widely recognized as a promising technique to avoid or even exploit ICI, that can enhance system throughput especially for cell-edge users [2]-[4].

The cooperative strategies of CoMP transmission include coordinated beamforming (CB) and joint processing (JP), depending on the information exchanged among the coordinated BSs. In CB, each BS transmits only signals intended for users in its own cell, but forms beams in such a way as to minimize interference to users in neighboring cells. In JP, BSs in a cooperative cluster jointly transmit signals to all users in the cluster. To implement centralized cooperative transmission, all BSs in the cooperative cluster are connected to a central unit (CU) through low-latency backhaul links to

This work was supported in part by the national key project of next generation wideband wireless communication networks (2011ZX03003-001) and the International S\&T Cooperation Program of China (ISCP) (2008DFA12100). share necessary information among the cooperated BSs. In the CoMP-CB transmission mode, only channel state information (CSI) is shared among BSs to facilitate each BS for designing individual precoding [5], [6]. In the CoMP-JP transmission mode, both data and CSI of all co-scheduled users in the cluster are shared among the cooperated BSs to design global precoding [2], [3], [7]. Since CoMP-CB avoids ICI while CoMP-JP converts the ICI into useful signal, CoMP-JP always outperforms CoMP-CB when the data and CSI are perfectly shared among the BSs [4].

In practice, the capacity of backhaul links in existing systems are limited, which imposes strict constraints on the data and CSI sharing for cooperative transmission, so that CoMP is not superior under all circumstances anymore. Compared with data sharing, the impact of CSI sharing on backhaul links is negligible [8]. A number of papers have suggested methods to optimize CoMP networks under backhaul constraints [9]-[12]. The capacity bounds for different cooperative schemes were obtained in [9]. The authors in [10] proposed an algorithm to select cooperative BSs for each mobile station aiming at maximizing the network utility subject to backhaul-capacity constraint. The strategy of rate splitting was studied in [11] to efficiently use the limited backhaul capacity, where each BS divides its data into a common part and a private part, and only the common data is shared among the BSs for joint transmission. The authors in [12] compared the performance of CoMP-CB and CoMP-JP transmission by simulations and showed that CoMP-CB may outperform CoMP-JP when the limited-capacity backhaul is taken into account.

In this paper, we propose a method to select the transmission mode between CoMP-CB and CoMP-JP modes to improve the performance of downlink CoMP system with limited-capacity backhaul. Specifically, we propose a closed-form decision rule for mode selection, which depends on local large-scale fading gains of all the users co-scheduled in the same time-frequency resource. With the transmission mode selection, partial users are served by JP while the others are served by $\mathrm{CB}$, therefore the mode selection system is superior to the system with a single, fixed, CoMP-CB or CoMP-JP mode.

The remainder of this paper is organized as follows. In Section II, we present the system model. In Section III, we analyze the average performance of CoMP-CB and CoMP-JP mode respectively and propose the transmission mode selec- 


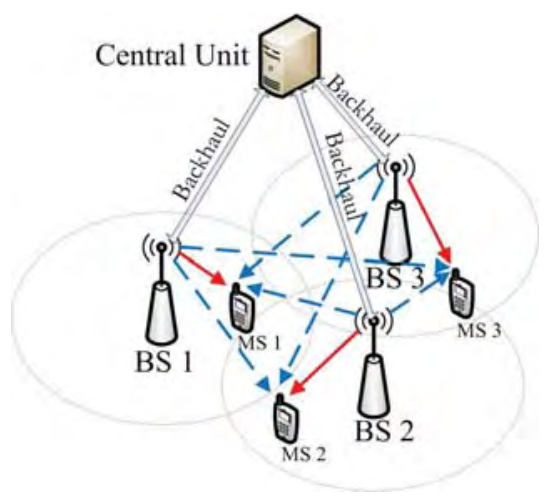

Fig. 1. An example of a three BSs cooperative cluster, where MS 1 is the local user of BS 1 .

tion method. Simulation and numerical results are provided in Section IV, followed by conclusions in Section V.

\section{SYSTEM MODEL}

Consider a cooperative cluster consisting of $B \mathrm{BSs}$, each equipped with $N_{t}$ antennas, while mobile stations (users) have single-antenna terminals. The assumption of single-antenna users is made for simplicity and does not preclude applying the results of this work to multiple antenna users. In CoMP$\mathrm{CB}$, one active single-antenna user is served by one BS, while in CoMP-JP, $B$ users are jointly served by the $B$ BSs with multi-user MIMO precoding.

We suppose that each $\mathrm{BS}$ is connected with the $\mathrm{CU}$ via a limited-capacity backhaul link, which is used to convey the downlink data and CSI from CU to each BS. The capacities of all backhaul links are all equal to $C$ bps/Hz. In the following, we ignore the burden caused by CSI sharing on the backhaul links [8], and only consider the impact of data sharing on the limited-capacity backhaul.

Denote by $\mathbf{g}_{i j} \in \mathbb{C}^{N_{t} \times 1}$ as the flat-fading small-scale channel states between BS $i$ and user $j$, each entry of which is complex Gaussian random variable with zero mean and unit variance, and all small-scale fading channel vectors are assumed as independent and identically distributed (i.i.d). Denote by $\mathbf{h}_{i j}=\alpha_{i j} \mathbf{g}_{i j}$ as the composite channel vector between BS $i$ and user $j$, and $\mathbf{h}_{j}=\left[\mathbf{h}_{1 j}^{H}, \ldots, \mathbf{h}_{B j}^{H}\right]^{H} \in \mathbb{C}^{B N_{t} \times 1}$ represents the global channel vector of user $j$, where $\alpha_{i j}$ is the large scale amplitude including path loss and shadowing. All the global channel vectors are assumed to be perfectly known at the CU.

\section{A. Coordinated Beamforming $(C B)$}

In the $\mathrm{CB}$ transmission mode, each BS obtains the data of its local user and the CSI of all active users in the cooperative cluster from the $\mathrm{CU}$ through the backhaul link, and then designs its precoder to avoid creating interference to other cells. The received signal at user $j$ can be expressed as

$$
y_{j}^{\mathrm{CB}}=\sqrt{p_{j}^{\mathrm{CB}}} \mathbf{h}_{j j}^{H} \mathbf{v}_{j j} x_{j}+\underbrace{\sum_{i \neq j}^{B} \sqrt{p_{i}^{\mathrm{CB}}} \mathbf{h}_{i j}^{H} \mathbf{v}_{i i} x_{i}}_{I C I}+z_{j},
$$

where $x_{j}$ is the transmit symbol for user $j$ with $\mathbf{E}\left\{\left|x_{j}\right|^{2}\right\}=1$, $\mathbf{v}_{j j} \in \mathbb{C}^{N_{t} \times 1}$ is the precoding vector at BS $j$ for user $j$ with $\left\|\mathbf{v}_{j j}\right\|^{2}=1, p_{j}^{\mathrm{CB}}$ is the transmit power allocated for user $j$, and $z_{j}$ is the Gaussian noise with variance $\sigma^{2}$.

To eliminate ICI completely, the precoding vector should satisfy $\mathbf{h}_{i j}^{H} \mathbf{v}_{i i}=0, \forall i \neq j .{ }^{1}$ Then the received signal-to-noiseand interference ratio (SINR) of user $j$ can be obtained as

$$
\begin{aligned}
\gamma_{j}^{\mathrm{CB}} & =\frac{p_{j}^{\mathrm{CB}}\left|\mathbf{h}_{j j}^{H} \mathbf{v}_{j j}\right|^{2}}{\sigma^{2}}=\frac{p_{j}^{\mathrm{CB}} \alpha_{j j}^{2}\left|\mathbf{g}_{j j}^{H} \mathbf{v}_{j j}\right|^{2}}{\sigma^{2}} \\
& =\frac{p_{j}^{\mathrm{CB}} \alpha_{j j}^{2}|| \mathbf{g}_{j j} \|^{2}\left|\tilde{\mathbf{g}}_{j j}^{H} \mathbf{v}_{j j}\right|^{2}}{\sigma^{2}},
\end{aligned}
$$

where $\tilde{\mathbf{g}}_{j j}$ represents the channel direction of $\mathbf{g}_{j j},\left|\tilde{\mathbf{g}}_{j j}\right|=1$. We consider per-user power constraint ${ }^{2}$ (PUPC) [15], which means that $p_{j}^{\mathrm{CB}} \leq 1, j=1, \ldots, B$ in (2).

\section{B. Joint Processing (JP)}

In the JP transmission mode, the data and CSI of all active users in the cluster are conveyed from the CU to each BS. The received signal at user $j$ is expressed as

$$
y_{j}^{\mathrm{JP}}=\sqrt{p_{j}^{\mathrm{JP}}} \mathbf{h}_{j}^{H} \mathbf{w}_{j} x_{j}+\sum_{i \neq j}^{B} \sqrt{p_{i}^{\mathrm{JP}}} \mathbf{h}_{j}^{H} \mathbf{w}_{i} x_{i}+z_{j},
$$

where $\mathbf{w}_{j} \in \mathbb{C}^{B N_{t} \times 1}$ is the global precoding vector for user $j$ with $\left\|\mathbf{w}_{j}\right\|^{2}=1$, and $p_{j}^{\mathrm{JP}}$ is the power allocated for user $j$. With PUPC, we have $p_{j}^{\mathrm{JP}} \leq 1$.

To completely eliminate interference we consider zeroforcing percoding [16], i.e., $\mathbf{h}_{j}^{H} \mathbf{w}_{i}=0, \forall i \neq j$. The received SINR of user $j$ and can be obtained as

$$
\gamma_{j}^{\mathrm{JP}}=\frac{p_{j}^{\mathrm{JP}}\left|\mathbf{h}_{j}^{H} \mathbf{w}_{j}\right|^{2}}{\sigma^{2}} .
$$

\section{Mode Switching Between COMP-CB AND COMP-JP}

We now come to the core of our paper, the development of a criterion for switching between the CB and JP. Our strategy is intended to provide a semi-dynamic mode selection that needs little signaling and is robust to the signaling latency [17]. We thus employ the average sum rate as the performance metric for mode selection instead of instantaneous sum rate.

We start by analyzing the average sum rate of CoMP-CB and JP transmission. Employing a mapping of capacity constraints onto equivalent power constraints, and by comparing their performance under limited-capacity backhaul, we derive

\footnotetext{
${ }^{1}$ We consider this precoder for analytical tractability. Precoders optimized for alternative criteria could be based, e.g., on minimization of mean-square error, or maximization of signal-to-leakage ratio [13, Chapter 23.3].

${ }^{2}$ The systems under different power constraints perform closely when the number of users is large [14].
} 
the criterion for mode selection method between CoMP-CB and JP.

\section{A. Average Sum Rate}

The average sum rate of CoMP-CB and JP transmission mode can respectively be expressed as follows,

$$
\begin{aligned}
& R_{\text {sum }}^{\mathrm{CB}}=\sum_{j=1}^{B} R_{j}^{\mathrm{CB}}=\sum_{j=1}^{B} \mathbf{E}\left\{\log _{2}\left(1+\gamma_{j}^{\mathrm{CB}}\right)\right\}, \\
& R_{\text {sum }}^{\mathrm{JP}}=\sum_{j=1}^{B} R_{j}^{\mathrm{JP}}=\sum_{j=1}^{B} \mathbf{E}\left\{\log _{2}\left(1+\gamma_{j}^{\mathrm{JP}}\right)\right\},
\end{aligned}
$$

where $R_{j}^{\mathrm{CB}}$ and $R_{j}^{\mathrm{JP}}$ respectively represent the average rate of user $j$ under CoMP-CB and JP mode, and $\mathbf{E}\{\cdot\}$ is the expectation over small-scale channel fading states.

We can use Jensen's inequality to obtain the upper bound of the average sum rates in (5) and (6) as

$$
\begin{aligned}
R_{\text {sum }}^{\mathrm{CB}} & \leq \sum_{j=1}^{B} \log _{2}\left(1+\mathbf{E}\left\{\gamma_{j}^{\mathrm{CB}}\right\}\right) \\
= & \sum_{j=1}^{B} \log _{2}\left(1+\frac{p_{j}^{\mathrm{CB}} \alpha_{j j}^{2} \cdot \mathbf{E}\left\{\left\|\mathbf{g}_{j j}\right\|^{2}\left|\tilde{\mathbf{g}}_{j j}^{H} \mathbf{v}_{j j}\right|^{2}\right\}}{\sigma^{2}}\right) \\
R_{\text {sum }}^{\mathrm{JP}} & \leq \sum_{j=1}^{B} \log _{2}\left(1+\mathbf{E}\left\{\gamma_{j}^{\mathrm{JP}}\right\}\right) \\
= & \sum_{j=1}^{B} \log _{2}\left(1+\frac{p_{j}^{\mathrm{JP}} \cdot \mathbf{E}\left\{\left|\mathbf{h}_{j}^{H} \mathbf{w}_{j}\right|^{2}\right\}}{\sigma^{2}}\right)
\end{aligned}
$$

It is not hard to show that the upper bounds are very tight when the number of antennas at each BS is large, since the variance of SINR in the $\log$ function becomes small for large $N_{t}$. Therefore, we employ the upper bounds of the average sum rate for analysis in the following, which leads to a closedform decision rule for mode switching.

Since user scheduling has a large impact on the performance, in the following we consider two different scheduling methods: random and orthogonal user scheduling, which correspond to the worst and best cases.

1) Random User Scheduling: With random userscheduling, user $j, j=1, \ldots, B$ are randomly selected from all the candidate users in the $B$ cells. This corresponds a scenario when there is only one candidate user randomly located in each cell.

In (7), the variable $\left\|\mathbf{g}_{j j}\right\|^{2}$ and $\left|\tilde{\mathbf{g}}_{j j}^{H} \mathbf{v}_{j j}\right|^{2}$ are independent since the norm and direction of a Gaussian uniform vector are independent. Therefore, $\mathbf{E}\left\{|| \mathbf{g}_{j j}||^{2}\left|\tilde{\mathbf{g}}_{j j}^{H} \mathbf{v}_{j j}\right|^{2}\right\}=\mathbf{E}\left\{\left\|\mathbf{g}_{j j}\right\|^{2}\right\}$. $\mathbf{E}\left\{\left|\tilde{\mathbf{g}}_{j j}^{H} \mathbf{v}_{j j}\right|^{2}\right\}=N_{t} \cdot \mathbf{E}\left\{\left|\tilde{\mathbf{g}}_{j j}^{H} \mathbf{v}_{j j}\right|^{2}\right\}$. Moreover, the variable $\left|\tilde{\mathbf{g}}_{j j}^{H} \mathbf{v}_{j j}\right|^{2}$ follows a beta distribution with parameters $B-1$ and $N_{t}-B+1$ when random scheduling is considered [18]. Applying the property of beta distributions, we have $\mathbf{E}\left\{\left|\tilde{\mathbf{g}}_{j j}^{H} \mathbf{v}_{j j}\right|^{2}\right\}=\left(N_{t}-B+1\right) / N_{t}$. Thus, the average sum rate of CoMP-CB with random user-scheduling can be obtained as

$$
R_{\text {sum }}^{\mathrm{CB}-\mathrm{R}}=\sum_{j=1}^{B} \log _{2}\left(1+\frac{\left(N_{t}-B+1\right) p_{j}^{\mathrm{CB}} \alpha_{j j}^{2}}{\sigma^{2}}\right) .
$$

In (8), the value of $\mathbf{E}\left\{\left|\mathbf{h}_{j}^{H} \mathbf{w}_{j}\right|^{2}\right\}$ with random scheduling has been obtained in [19] as

$$
\mathbf{E}\left\{\left|\mathbf{h}_{j}^{H} \mathbf{w}_{j}\right|^{2}\right\}=N_{t} \sum_{i=1}^{B} \eta_{i} \alpha_{i j}^{2},
$$

where $\eta_{i}, i=1, \ldots, B$ is determined by the following equations,

$$
\eta_{i}=1-\frac{B-1}{B N_{t}} \sum_{j=1}^{B} \frac{\eta_{i} \alpha_{i j}^{2}}{\sum_{l=1}^{B} \eta_{l} \alpha_{l j}^{2}} .
$$

The value of $\eta_{i}$ depends on the large-scale fading gains of all $B$ users, and $\sum_{i=1}^{B} \eta_{i}=\left(B N_{t}-B+1\right) / N_{t}$. Then, the average sum rate of CoMP-JP transmission with random userscheduling can be obtained as

$$
R_{\text {sum }}^{\mathrm{JP}-\mathrm{R}}=\sum_{j=1}^{B} \log _{2}\left(1+\frac{N_{t} \cdot p_{j}^{\mathrm{JP}} \sum_{i=1}^{B} \eta_{i} \alpha_{i j}^{2}}{\sigma^{2}}\right) .
$$

2) Orthogonal User-Scheduling: With orthogonal scheduling, the users $j, j=1, \ldots, B$, are selected perfectly orthogonal to each other. This can be achieved when there are enough candidate users in each cell.

In the CoMP-CB mode, we schedule users that satisfy $\mathbf{g}_{j j}^{H} \mathbf{g}_{j i}=0, i, j=1, \ldots, B, i \neq j$. Therefore, the precoding vector at BS $j$ equals the direction of $\mathbf{g}_{j j}$, i.e., $\mathbf{v}_{j j}=\tilde{\mathbf{g}}_{j j}$, and $\left|\tilde{\mathbf{g}}_{j j}^{H} \mathbf{v}_{j j}\right|^{2}=1$. The average sum rate of CoMP-CB in (7) with orthogonal scheduling is derived as

$$
R_{\text {sum }}^{\mathrm{CB}-\mathrm{O}}=\sum_{j=1}^{B} \log _{2}\left(1+\frac{N_{t} \cdot p_{j}^{\mathrm{CB}} \alpha_{j j}^{2}}{\sigma^{2}}\right) .
$$

In the CoMP-JP mode, we schedule users that satisfy $\mathbf{h}_{i}^{H} \mathbf{h}_{j}=0, i \neq j$. Therefore, the global precoding vector $\mathbf{w}_{j}=\mathbf{h}_{j} /\left\|\mathbf{h}_{j}\right\|$. Then $\mathbf{E}\left\{\left|\mathbf{h}_{j}^{H} \mathbf{w}_{j}\right|\right\}=\mathbf{E}\left\{\left\|\mathbf{h}_{j}\right\|^{2}\right\}=$ $N_{t} \sum_{i=1}^{B} \alpha_{i j}^{2}$. The average sum rate of CoMP-JP in (8) with orthogonal scheduling is derived as

$$
R_{\text {sum }}^{\mathrm{JP}-\mathrm{O}}=\sum_{j=1}^{B} \log _{2}\left(1+\frac{N_{t} \cdot p_{j}^{\mathrm{JP}} \sum_{i=1}^{B} \alpha_{i j}^{2}}{\sigma^{2}}\right) .
$$

\section{B. Limited-Capacity Backhaul}

The downlink user date rate will be restricted by the limitedcapacity backhaul links between the CU and BSs.

In the CoMP-CB mode, the CU only conveys the downlink data of local user to each BS through backhaul link. Thus, the backhaul constraint is,

$$
R_{j}^{\mathrm{CB}} \leq C
$$

In the CoMP-JP mode, the CU conveys the downlink data of all active users to each BS. Thus, the backhaul constraint 
is,

$$
R_{\text {sum }}^{\mathrm{JP}} \leq C .
$$

The constraints in (15) and (16) can translate to the constraints on the permitted transmit powers $p_{j}^{\mathrm{CB}}$ and $p_{j}^{\mathrm{JP}}, j=$ $1, \ldots, B$. In other words, there is no point in achieving a higher over-the-air capacity (with its associated transmit power) than the backhaul capacity can provide. Substituting (9) (12) (13) (14) into (15) and (16), we obtain that

$$
\left\{\begin{array}{l}
\left(1+\Gamma_{j}^{\mathrm{CB}} \cdot p_{j}^{\mathrm{CB}}\right) \leq 2^{C}, \\
\prod_{j=1}^{B}\left(1+\Gamma_{j}^{\mathrm{JP}} \cdot p_{j}^{\mathrm{JP}}\right) \leq 2^{C},
\end{array}\right.
$$

where $\Gamma_{j}^{\mathrm{CB}}=\left(N_{t}-B+1\right) \alpha_{j j}^{2} / \sigma^{2}, \Gamma_{j}^{\mathrm{JP}}=N_{t} \sum_{i=1}^{B} \eta_{i} \alpha_{i j}^{2} / \sigma^{2}$ under random user scheduling, and $\Gamma_{j}^{\mathrm{CB}}=N_{t} \alpha_{j j}^{2} / \sigma^{2}, \Gamma_{j}^{\mathrm{JP}}=$ $N_{t} \sum_{i=1}^{B} \alpha_{i j}^{2} / \sigma^{2}$ under orthogonal scheduling. A second constraint of the transmit power $p_{j}^{\mathrm{CB}}$ and $p_{j}^{\mathrm{JP}}$ comes from the PUPC limits (which is 1 per assumption):

$$
p_{j}^{\mathrm{CB}} \leq 1, \quad p_{j}^{\mathrm{JP}} \leq 1 .
$$

For user $k$, the backhaul constraint on $p_{k}^{\mathrm{CB}}$ and $p_{k}^{\mathrm{JP}}$ in (17) can be transformed into

$$
\begin{aligned}
& p_{k}^{\mathrm{CB}} \leq \frac{2^{C}-1}{\Gamma_{k}^{\mathrm{CB}}} \triangleq T_{k}^{\mathrm{CB}}, \\
& p_{k}^{\mathrm{JP}} \leq \frac{2^{C} / \prod_{j \neq k}^{B}\left(1+\Gamma_{k}^{\mathrm{JP}} \cdot p_{k}^{\mathrm{JP}}\right)-1}{\Gamma_{k}^{\mathrm{JP}}} \triangleq T_{k}^{\mathrm{JP}} .
\end{aligned}
$$

Since $T_{k}^{\mathrm{CB}}>T_{k}^{\mathrm{JP}}$, the limited-capacity backhaul imposes much rigid constraint on $p_{j}^{\mathrm{JP}}$ than on $p_{j}^{\mathrm{CB}}$. To compare the maximum value of $R_{\text {sum }}^{\mathrm{CB}}$ and $R_{\text {sum }}^{\mathrm{JP}}$ under the limited backhaul capacity, in the following we consider three cases according to the dominant constraints of $p_{j}^{\mathrm{CB}}$ and $p_{j}^{\mathrm{JP}}$.

1) All $p_{j}^{\mathrm{CB}}$ and all $p_{j}^{\mathrm{JP}}, j=1, \ldots, B$, are constrained by maximal transmit power.

In this case, the value of $R_{\text {sum }}^{\mathrm{CB}}$ and $R_{\text {sum }}^{\mathrm{JP}}$ are maximized when $p_{j}^{C B}=p_{j}^{J P}=1, j=1, \ldots, B$. There,

$$
\begin{aligned}
& R_{\text {sum }}^{\mathrm{CB} *}=\sum_{j=1}^{B} \log _{2}\left(1+\Gamma_{j}^{\mathrm{CB}}\right), \\
& R_{\text {sum }}^{\mathrm{JP} *}=\sum_{j=1}^{B} \log _{2}\left(1+\Gamma_{j}^{\mathrm{JP}}\right) .
\end{aligned}
$$

Since $\Gamma_{j}^{\mathrm{JP}}>\Gamma_{j}^{\mathrm{CB}}$, the average sum rate of CoMP-JP mode always exceeds that of CoMP-CB mode in this case.

2) At least one of $p_{j}^{\mathrm{CB}}, j=1, \ldots, B$, is constrained by the limited backhaul, and all $p_{j}^{\mathrm{JP}}, j=1, \ldots, B$, are constrained by the limited backhaul.

In this case, the maximum value of $R_{\text {sum }}^{\mathrm{JP}}$ equals to the backhaul capacity $C$, and the maximum value of $R_{\text {sum }}^{\mathrm{CB}}$ exceeds $C$ since at least one of $R_{j}^{\mathrm{CB}}$ equals to $C$. Therefore, the average sum rate of CoMP-CB is always higher than that of CoMP-JP.

3) All $p_{j}^{\mathrm{CB}}, j=1, \ldots, B$, are constrained by the maximal transmit power, and all $p_{j}^{\mathrm{JP}}, j=1, \ldots, B$, are constrained by the limited backhaul. In this case, $R_{\text {sum }}^{\mathrm{JP} *}=C, p_{j}^{\mathrm{CB}}=1, j=1, \ldots, B$ and $R_{\text {sum }}^{\mathrm{CB} *}=\sum_{j=1}^{B} \log _{2}\left(1+\Gamma_{j}^{\mathrm{CB}}\right)$.

We choose the CoMP-JP mode if $\sum_{j=1}^{B} \log _{2}\left(1+\Gamma_{j}^{C B}\right)<C$, which can be rewritten as

$$
\prod_{j=1}^{B}\left(1+\Phi \cdot \alpha_{j j}^{2}\right)<2^{C}
$$

where $\Phi=\left(N_{t}-B+1\right) / \sigma^{2}$ under random user scheduling, and $\Phi=N_{t} / \sigma^{2}$ under orthogonal user scheduling. Otherwise, we choose the CoMP-CB mode if

$$
\prod_{j=1}^{B}\left(1+\Phi \cdot \alpha_{j j}^{2}\right) \geq 2^{C} .
$$

From above analysis we can see that transmission mode selection is needed only for the third case, where the mode selection rule is expressed as (21) and (22). In the first case, $R_{\text {sum }}^{\mathrm{CB} *}<R_{\text {sum }}^{\mathrm{JP} *} \leq C$ always holds. This implies that if a group of users satisfy the inequality of (21), they belong to the first case. Similarly, in the second case, $\sum_{j=1}^{B} \log _{2}\left(1+\Gamma_{j}^{C B}\right) \geq$ $R_{\text {sum }}^{\mathrm{CB} *}>C$ always holds. This means that if a group of users satisfy the inequality of (22), they belong to the second case. That is to say, (21) and (22) can be employed as a unified decision rule for the CoMP mode selection in all cases.

Several observations can be obtained as follows.

- The decision rule for CoMP transmission mode selection depends on many factors, such as the large-scale fading gains, the noise power, the number of antennas at each BS, and the capacity of backhaul links.

- The large-scale fading gains of all active users jointly determine the mode selection rule. In other words, the transmission mode depends on the location of all the coscheduled users. This implies that we can not obtain a unique distance threshold (or SINR threshold) to divide the active users into two groups that are respectively served by CoMP-JP and CoMP-CB transmission mode.

- By design, only the large-scale channel characteristics, but not the instantaneous fading realizations, between each BS and its local user affect the mode selection rule. This indicates that the proposed transmission mode selection method requires little channel information and works in a semi-dynamic way.

\section{Simulation And Numerical Results}

In this section, we will validate our analysis and evaluate the performance of the proposed mode switching method via simulation and numerical results.

We consider a cellular network where the radius of each cell is $250 \mathrm{~m}$, and the minimum distance from all users to each BS is $35 \mathrm{~m}$. The path loss follows a power law with decay exponent factor is set as 3.76. The edge SNR is defined similarly as in [20], which reflects the strength of the noise power as well as the inter-cluster interference. The capacity of 
backhaul links in practical systems is in a range of $100 \mathrm{Mbps}$ $\sim 2$ Gbps. Therefore we consider $C \sim[1 \mathrm{bps} / \mathrm{Hz}, 20 \mathrm{bps} / \mathrm{Hz}]$ with $100 \mathrm{MHz}$ system bandwidth [21].

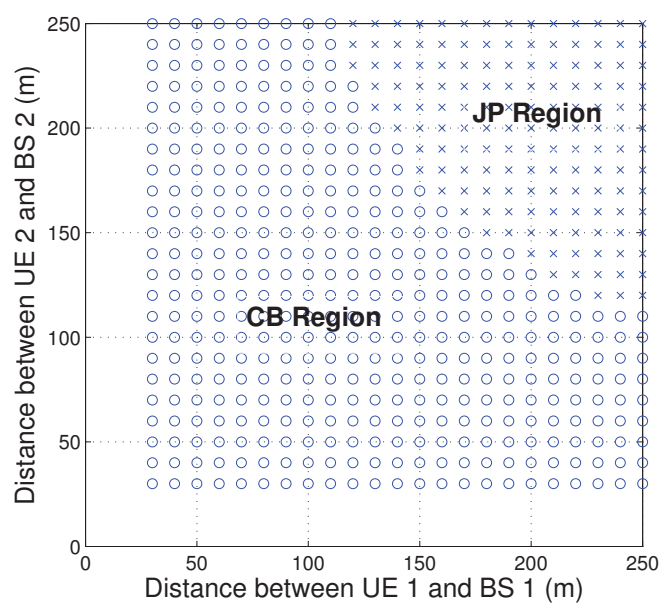

(a) CoMP-JP and CB mode regions under random user scheduling, where $N_{t}=4, C=8 \mathrm{bps} / \mathrm{Hz}$ and edge $\mathrm{SNR}=0 \mathrm{~dB}$.

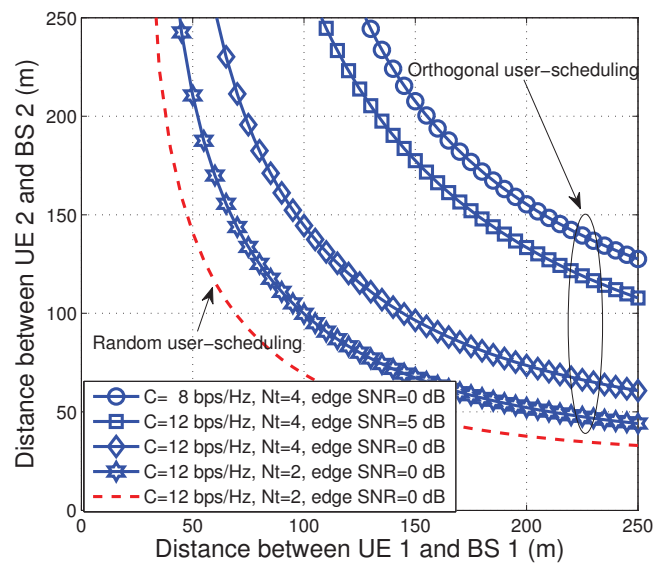

(b) The border of CoMP JP region and CB region

Fig. 2. The results of mode selection in a two BSs cooperation scenario.

In Fig. 2, we consider a two BSs cooperation scenario, i.e., $B=2$, and two users are dropped on the line connecting BS 1 and BS 2. The region of CoMP-JP and CB modes numerically obtained from (21) and (22) are shown in Fig. 2(a), where $N_{t}=4, C=8 \mathrm{bps} / \mathrm{Hz}$ and edge $\mathrm{SNR}=0 \mathrm{~dB}$. We can see that the mode selection finally leads to two complementary regions of CoMP-CB and JP modes. When the two users are all located at the cell edge, CoMP-JP mode is selected. When one of the users is located at the cell center, CoMP-CB mode is selected. Note that boundary of the two regions does not mean a single distance or SINR threshold. Instead, it indicates that the mode selection results depend on the location of the co-scheduled two users. In Fig. 2(b), we show the border of CoMP-JP region and CoMP-CB region under different system parameters. We can see that as the capacity of backhaul links increases, the

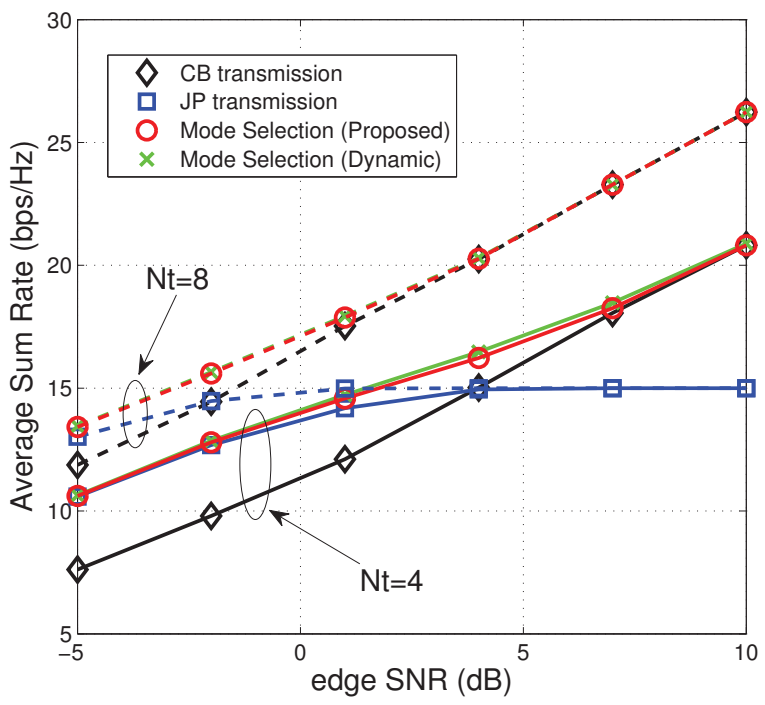

Fig. 3. Average sum rate comparison when $B=3$ and $C=15 \mathrm{bps} / \mathrm{Hz}$.

CoMP-JP region becomes larger. The higher edge SNR leads to a larger CoMP-CB region, and more antennas at each BS leads to a larger JP region. Orthogonal user scheduling leads to smaller CoMP-JP region compared with the random user scheduling.

In Fig. 3, we consider a three BSs cooperation scenario and compare the average sum rate of different systems when the capacity of each backhaul link is set as $15 \mathrm{bps} / \mathrm{Hz}$. The simulation results are obtained from 10000 independent drops of users, each of which has 1000 time slots with independent Rayleigh fading. The performance of purely CoMP-JP transmission outperforms that of purely CoMP-CB transmission in the low edge-SNR regime. The single CoMP-CB mode outperforms the single CoMP-JP mode in high edge-SNR regime, since the average sum rate of CoMP-JP is strictly restricted by the limited backhaul capacity. By selecting transmission modes between CoMP-CB and CoMP-JP, the system performance approaches to that of CoMP-JP at low edge SNR and approaches to that of CoMP-CB at high edge SNR. The proposed mode selection scheme outperforms both the single CoMP-CB mode and the single CoMP-JP mode at the medium edge SNRs.

In Fig. 4, we again consider the three BSs cooperation scenario and compare the average sum rate of different systems versus backhaul capacity constraints vias simulations, where the edge SNR is set to $0 \mathrm{~dB}$. As expected, the performance of CoMP-JP outperforms CoMP-CB at high backhaul capacity region, while CoMP-CB outperforms CoMP JP at low backhaul capacity region. By selecting transmission modes between CoMP-CB and JP, the performance approaches to that of CoMP-JP at high backhaul capacity region and approaches to that of CoMP-CB at low backhaul capacity region.

To show the impact of the backhaul constraint imposed on the average per-user rate shown in (15) and (16), we compare 


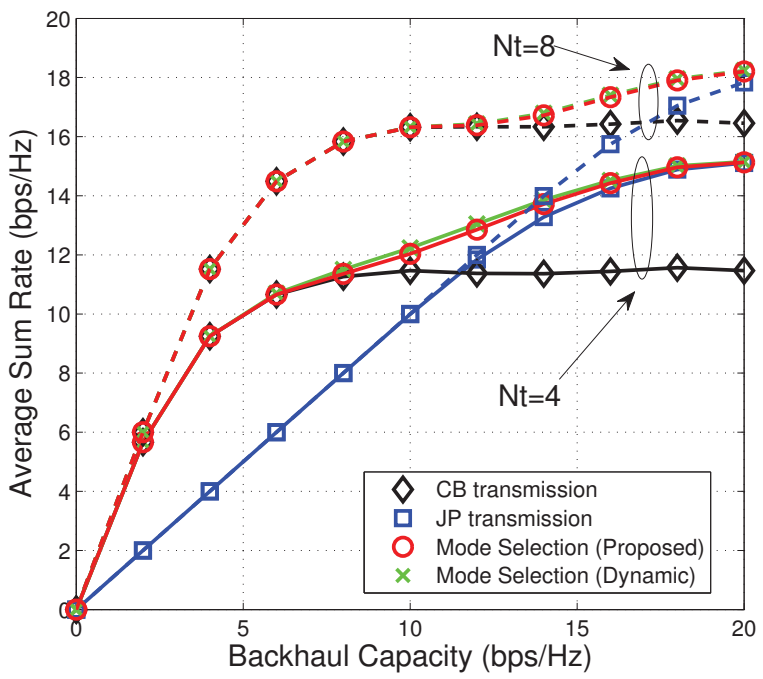

Fig. 4. Average sum rate comparison when $B=3$ and edge SNR is $0 \mathrm{~dB}$.

the proposed semi-dynamic mode selection scheme with that of a dynamic mode selection (the dash lines in Fig. 3 and Fig. 4) where the transmission mode is selected based on instantaneous channels, i.e., the performance metric is the instantaneous sum rate. The results show that the performance gap between the semi-dynamic and dynamic mode selection is minor. This indicates that the proposed semi-dynamic mode selection method can reduce the required signaling and control overhead, while providing nearly the same performance as dynamic mode selection. We also evaluate the tightness of the Jensen's inequity used to obtain (7) and (8), which results almost overlap with those with legend "CB transmission" and "JP transmission".

\section{CONCLUSION}

In this paper, a transmission mode selection method between CoMP-CB and CoMP-JP transmission was proposed under limited-capacity backhaul. We developed a closed-form decision rule for mode selection, which depends on the backhaul capacity, system configurations, noise power, as well as user scheduling. The proposed transmission mode selection method only requires the large-scale fading gains of local channels, which can operate in a semi-dynamic manner and require little signalling overhead. Simulation results confirm that the proposed method outperforms both the single CoMP-CB mode and single CoMP-JP mode transmission.

\section{REFERENCES}

[1] 3GPP TR 36.819 v0.0.1, "Coordinated multi-point operation for LTE physical layer aspects (Release 11)," Feb. 2011. [Online]. Available: ftp.3gpp.org

[2] A. F. Molisch, Internal Memo, AT\&T Labs - Research, 2001. Also see A F. Molisch, M. V. Clark, H. Dai, M. Z. Win, and J. H. Winters, "Method and apparatus for reducing interference in multiple-input-multiple-output (MIMO) systems," US Patent 7,912,014 (filed 2002, granted 2011).
[3] M. K. Karakayali, G. J. Foschini, and R. A. Valenzuela, "Network coordination for spectrally efficient communications in cellular systems," IEEE Wireless Commun. Mag., vol. 13, pp. 56-61, Aug. 2006.

[4] D. Gesbert, S. Hanly, H. Huang, S. Shamai, O. Simeone, and W. Yu, "Multi-cell MIMO cooperative networks: A new look at interference," IEEE J. Sel. Areas Commun., vol. 28, no. 9, pp. 1380-1408, Dec 2010.

[5] C. B. Chae, S. Kim, and R. W. Heath, "Network coordinated beamforming for cell-boundary users: Linear and nonlinear approaches," IEEE J. Select. Topics in Signal Processing, vol. 3, pp. 1094-1105, Dec. 2009.

[6] H. Dahrouj and W. Yu, "Coordinated beamforming for the multicell multi-antenna wireless system," IEEE Trans. Wireless Commun., vol. 9 pp. 1748-1759, May 2010 .

[7] A. Tölli , and M. Codreanu and M. Juntti, "Cooperative MIMO-OFDM cellular system with soft handover between distributed base station antennas," IEEE Trans. Wireless Commun., vol. 7, pp. 1428-1440, Apr. 2008 .

[8] D. Samardzija and H. Huang, "Determining backhaul bandwidth requirements for network MIMO," in Proc. Euro. Signal Processing Conf. (EUSIPCO), 2009.

[9] P. Marsch and G. Fettweis, "On downlink network MIMO under a constrained backhaul and imperfect channel knowledge," in Proc. IEEE GlobeCom, 2009.

[10] A. Chowdhery, W. Yu, and J. M. Cioffi, "Cooperative wireless multicell OFDMA network with backhaul capacity constraints," in Proc. IEEE ICC, 2011.

[11] R. Zakhour and D. Gesbert, "Optimized data sharing in multicell MIMO with finite backhaul capacity," to appear in IEEE Trans. Signal Processing, 2011.

[12] N. Seifi, M. Viberg, R. W. Heath, J. Zhang, and M. Coldrey, "Coordinated single-cell vs multi-cell transmission with limited-capacity backhaul," in Proc. IEEE ACSSC, 2010.

[13] A. F. Molisch, Wireless Communications. John Wiley Press, 2011.

[14] J. Zhang, R. Chen, J. G. Andrews, A. Ghosh, and R. W. Heath, "Network MIMO with clustered linear precoding," IEEE Trans. Wireless Commun. vol. 8, pp. 1910-1921, Apr. 2009

[15] H. Zhang, N. B. Mehta, A. F. Molisch, J. Zhang, and H. Dai, "Asynchronous interference mitigation in cooperative base station systems," IEEE Trans. Wireless Commun., vol. 7, pp. 155-165, Jan. 2008.

[16] T. Yoo and A. Goldsmith, "On the optimality of multiantenna broadcast scheduling using zero-forcing beamforming," IEEE J. Select. Areas Commun., vol. 24, pp. 528-541, Mar. 2006.

[17] P. Marsch and G. P. Fettweis, Coordinated Multi-Point in Mobile Communications: From Theory to Practice. Cambridge University Press, 2011.

[18] Q. Zhang and C. Yang, "Semi-dynamic mode selection in base station cooperative transmission system," in Proc. IEEE VTC Fall, 2011.

[19] H. Huh, A. Tulino, and G. Caire, "Network MIMO large-system analysis and the impact of CSIT estimation," in Proc. IEEE CISS, 2010.

[20] A. Papadogiannis, H. J. Bang, D. Gesbert, and E. hardouin, "Efficient selective feedback design for multicell cooperative networks," IEEE Trans. Veh. Technol., vol. 60, pp. 196-205, Jan. 2011.

[21] O. Tipmongkolsilp, S. Zaghloul, and A. Jukan, "The evolution of cellula backhaul technologies: Current issues and future trends," IEEE Commun. Surveys Tuts., vol. 13, pp. 97-113, 2011. 